\title{
HOMOTOPY DECOMPOSITION OF A SUSPENDED REAL TORIC SPACE
}

\author{
SUYOUNG CHOI, SHIZUO KAJI, AND STEPHEN THERIAULT
}

In memory of Professor Samuel Gitler

\begin{abstract}
We give $p$-local homotopy decompositions of the suspensions of real toric spaces for odd primes $p$. Our decomposition is compatible with the one given by Bahri, Bendersky, Cohen, and Gitler for the suspension of the corresponding real moment-angle complex, or more generally, the polyhedral product. As an application, we obtain a stable rigidity property for real toric spaces. This is a generalized version of the published paper 7 .
\end{abstract}

\section{INTRODUCTION}

For a simplicial complex $K$ on $m$-vertices $[m]=\{1, \ldots, m\}$ the real moment-angle complex $\mathbb{R} \mathcal{Z}_{K}$ (or the polyhedral product $\left.\left(\underline{D^{1}}, \underline{S^{0}}\right)^{K}\right)$ of $K$ is defined as follows:

$$
\begin{aligned}
\mathbb{R} \mathcal{Z}_{K} & =\left(\underline{D^{1}}, \underline{S^{0}}\right)^{K} \\
& :=\bigcup_{\sigma \in K}\left\{\left(x_{1}, \ldots, x_{m}\right) \in\left(D^{1}\right)^{m} \mid x_{i} \in S^{0} \text { when } i \notin \sigma\right\},
\end{aligned}
$$

where $D^{1}=[0,1]$ is the unit interval and $S^{0}=\{0,1\}$ is its boundary. It should be noted that $\mathbb{R} \mathcal{Z}_{K}$ is a topological manifold if $K$ is a simplicial sphere [3, Lemma 6.13].

There is a canonical $\mathbb{F}_{2}^{m}$-action on $\mathbb{R}_{K}$ which comes from the $\mathbb{F}_{2}$-action on the pair $\left(D^{1}, S^{0}\right)$. Any subgroup of $\mathbb{F}_{2}^{m}$ is specified as the kernel of a linear map $\lambda: \mathbb{F}_{2}^{m} \rightarrow \mathbb{F}_{2}^{n}$ for some $n \leq m$. The quotient space $\mathbb{R} \mathcal{Z}_{K} /$ ker $\lambda$ is denoted by $M(K, \lambda)$ and called the real toric space associated to $K$ and $\lambda$. We denote the $i$-th column of $\lambda$ by $\lambda(i)$.

Real toric spaces are a generalization to well-studied classes of spaces. When $\lambda$ satisfies the following condition, it is called a (mod 2) characteristic function of $K$ :

$$
\lambda\left(i_{1}\right), \ldots, \lambda\left(i_{\ell}\right) \text { are linearly independent in } \mathbb{F}_{2}^{n} \text { if }\left\{i_{1}, \ldots, i_{\ell}\right\} \in K \text {. }
$$

Lemma 1.1. The action of $\operatorname{ker} \lambda$ on $\mathbb{R} \mathcal{Z}_{K}$ is free if and only if (11) is satisfied.

Proof. Let $x=\left(x_{1}, x_{2}, \ldots, x_{m}\right) \in \mathbb{R}_{K}=\left(\underline{D^{1}}, \underline{S^{0}}\right)^{K}$ be the fixed point of an element $g=$ $\left(g_{1}, g_{2}, \ldots, g_{m}\right)^{t} \in \operatorname{ker} \lambda \subset \mathbb{F}_{2}^{m}$. This means either $g_{i}=0$ or $x_{i} \in\left(D^{1}\right)^{\mathbb{F}_{2}}=\{1 / 2\}$ for all $i \in[m]$. Let $\sigma \in K$ be a simplex such that $x \in\left(\underline{D^{1}}, \underline{S^{0}}\right)^{\sigma}$ and $\lambda_{\sigma}$ be the sub-matrix of $\lambda$ consisting of columns corresponding to $\sigma$. Let $g_{\sigma}$ be the sub-vector of $g$ corresponding to $\sigma$. Since $g \in \operatorname{ker} \lambda$, we have

$$
\lambda g=\lambda_{\sigma} g_{\sigma}+\lambda_{[m] \backslash \sigma} g_{[m] \backslash \sigma}=0 .
$$

Since $\mathbb{F}_{2}$ acts on $S^{0}$ freely, we have $g_{i}=0$ for $i \notin \sigma$. Then, by the previous equation we have $\lambda_{\sigma} g_{\sigma}=0$. Therefore, the condition (11) implies $g_{\sigma}=0$ and $g=0$. On the other hand, if (11) does not hold for a simplex $\sigma \in K$, the point $x_{i}=\left\{\begin{array}{ll}1 / 2 & (i \in \sigma) \\ 0 & (i \notin \sigma)\end{array}\right.$ is fixed by an element in $\operatorname{ker} \lambda$.

Date: August 24, 2018.

2010 Mathematics Subject Classification. Primary 55P15; Secondary 57S17.

Key words and phrases. homotopy decomposition, real toric manifold, real toric spaces, small cover.

The first named author was supported by Basic Science Research Program through the National Research Foundation of Korea(NRF) funded by the Ministry of Education(NRF-2011-0024975).

The second named author was partially supported by KAKENHI, Grant-in-Aid for Young Scientists (B) 26800043 and JSPS Postdoctoral Fellowships for Research Abroad. 
For a characteristic function $\lambda$, the corresponding real toric space $M(K, \lambda)$ is known as a small cover [10] if $K$ is a polytopal $(n-1)$-sphere, and as a real topological toric manifold [12] if $K$ is a star-shaped $(n-1)$-sphere.

In [1, Theorem 2.21] it is shown that there is a homotopy equivalence

$$
\Sigma \mathbb{R} \mathcal{Z}_{K} \simeq \Sigma \bigvee_{I \in[m]} \Sigma\left|K_{I}\right| \simeq \Sigma \bigvee_{I \notin K} \Sigma\left|K_{I}\right|
$$

where $K_{I}$ is the full subcomplex of $K$ on the vertex set $I$ and $\left|K_{I}\right|$ is its geometric realization so that $\left|K_{I}\right|$ is contractible when $I \in K$. In this short note, we give an analogous odd primary decomposition of the suspension of $M(K, \lambda)$.

Theorem 1.2. Let $M(K, \lambda)$ be a real toric space. Localized at an odd prime $p$ or the rationals (denoted by $p=0$ ) there is a homotopy equivalence

$$
\Sigma(M(K, \lambda)) \simeq_{p} \Sigma \bigvee_{I \in \operatorname{Row}(\lambda)} \Sigma\left|K_{I}\right|
$$

where $\operatorname{Row}(\lambda)$ is the space of $m$-dimensional $\mathbb{F}_{2}$-vectors spanned by the rows of $\lambda$ associated to $\lambda$. We identify vectors in $\mathbb{F}_{2}^{m}$ with subsets of $[m]$ in the obvious manner.

Remark 1.3. When $\lambda$ is the identity matrix, $M(K, \lambda)$ is nothing but $\mathbb{R} \mathcal{Z}_{K}$. On the other hand, when $\lambda$ is zero, $M(K, \lambda)$ is the cone over $|K|$ and contractible.

The restriction to odd primes arises because when $|\operatorname{ker} \lambda|$ is inverted in a coefficient ring $R$ then the quotient map $\mathbb{R} \mathcal{Z}_{K} \longrightarrow M(K, \lambda)$ induces an injection in cohomology with image the invariant subring $H^{*}\left(\mathbb{R} \mathcal{Z}_{K} ; R\right)^{\mathrm{ker} \lambda}$ (see, for example, [2, §III.2]). This will be used to help analyze the topology of $M(K, \lambda)$. As ker $\lambda$ has order a power of 2 we can take $R$ to be $\mathbb{Z}_{(p)}$ or $\mathbb{Q}$. In fact, Theorem 1.2 fails when $p=2$ in simple cases. For example, if $K$ is the boundary of a triangle and $\lambda=\left(\begin{array}{lll}1 & 0 & 1 \\ 0 & 1 & 1\end{array}\right)$, then $M(K, \lambda)=\mathbb{R} P^{2}$ but each $\Sigma\left|K_{I}\right|$ is contractible.

Recent work of $\mathrm{Yu}$ [14] gave a different decomposition of the suspension of certain quotient spaces of $\mathbb{R} \mathcal{Z}_{K}$. He considers $\lambda$ which is associated to a partition on the vertices of $K$, and proves that $M(K, \lambda)$ decomposes analogously to the Bahri, Bendersky, Cohen and Gitler decomposition. Yu's decomposition has the advantage of working integrally, but it has the disadvantage of working only for particular $\lambda$. Our decomposition, by contrast, works only after localizing at an odd prime but holds for any $\lambda$.

Acknowledgement. The authors would like to thank Daisuke Kishimoto for pointing out an error in an earlier version of the paper, and Mikiya Masuda for pointing out that our argument works for not only characteristic functions but also any linear maps $\lambda$.

\section{Polyhedral PRoduct ANd its Stable DeCOMposition}

Let us first recall Bahri, Bendersky, Cohen and Gitler's argument in [1]. To make it more clear, we present it in its full polyhedral product form. Let $K$ be a simplicial complex on the vertex set $[m]$ and for $1 \leq i \leq m$ let $\left(X_{i}, A_{i}\right)$ be pairs of pointed $C W$-complexes. If $\sigma$ is a face of $K$ let

$$
(\underline{X}, \underline{A})^{\sigma}=\prod_{i=1}^{m} Y_{i} \quad \text { where } \quad Y_{i}=\left\{\begin{array}{cc}
X_{i} & \text { if } i \in K \\
A_{i} & \text { if } i \notin K .
\end{array}\right.
$$

The polyhedral product is

$$
(\underline{X}, \underline{A})^{K}=\bigcup_{\sigma \in K}(\underline{X}, \underline{A})^{\sigma} .
$$

Notice that $(\underline{X}, \underline{A})^{K}$ is a subspace of the product $\prod_{i=1}^{m} X_{i}$. There is a canonical quotient map from the product to the smash product, $\prod_{i=1}^{m} X_{i} \longrightarrow \bigwedge_{i=1}^{m} X_{i}$. The smash polyhedral product 
$\widehat{(\underline{X}, \underline{A}}^{K}$ is the image of the composite $(\underline{X}, \underline{A})^{K} \longrightarrow \prod_{i=1}^{m} X_{i} \rightarrow \bigwedge_{i=1}^{m} X_{i}$. In particular, mapping onto the image gives a map $(\underline{X}, \underline{A})^{K} \longrightarrow \widehat{(\underline{X}, \underline{A}}^{K}$.

Let $I \subset[m]$. As in [11, 2.2.3(i)], projecting $\prod_{i=1}^{m} X_{i}$ onto $\prod_{i \in I} X_{i}$ induces a map of polyhedral products $(\underline{X}, \underline{A})^{K} \longrightarrow(\underline{X}, \underline{A})^{K_{I}}$. We then obtain a composition into a smash polyhedral product:

$$
p_{I}:(\underline{X}, \underline{A})^{K} \longrightarrow(\underline{X}, \underline{A})^{K_{I}} \longrightarrow \widehat{(\underline{X}, \underline{A}}^{K_{I}} .
$$

Suspending, we can add every such composition over all full subcomplexes of $K$, giving a composition

$$
\bar{H}: \Sigma(\underline{X}, \underline{A})^{K \stackrel{c o m u l}{\longrightarrow}} \bigvee_{I \subset[m]} \Sigma(\underline{X}, \underline{A})^{K_{I}} \stackrel{\vee \Sigma p_{I}}{\longrightarrow} \bigvee_{I \subset[m]} \Sigma(\underline{X}, \underline{A})^{K_{I}} .
$$

Bahri, Bendersky, Cohen and Gitler [1, Theorem 2.10] show that $\bar{H}$ is a homotopy equivalence.

Further, in the special case when each $X_{i}$ is contractible, they show that there is a homotopy equivalence $\widehat{(\underline{X}, \underline{A}}^{K_{I}} \simeq \Sigma\left(\left|K_{I}\right| \wedge \widehat{A}^{I}\right)$ [1, Theorem 2.19], where $\widehat{A}^{I}=\bigwedge_{j=1}^{k} A_{i_{j}}$ for $I=\left(i_{1}, \ldots, i_{k}\right)$. Consequently, when each $X_{i}$ is contractible the map $\bar{H}$ specializes to a homotopy equivalence

$$
H: \Sigma(\underline{X}, \underline{A})^{K} \longrightarrow \bigvee_{I \subset[m]} \Sigma(\underline{X}, \underline{A})^{K_{I}} \longrightarrow \bigvee_{I \subset[m]} \Sigma(\underline{\underline{X}, \underline{A}})^{K_{I}} \stackrel{\simeq}{\longrightarrow} \bigvee_{I \subset[m]} \Sigma^{2}\left(\left|K_{I}\right| \wedge \widehat{A}^{I}\right) .
$$

In our case, each pair $\left(X_{i}, A_{i}\right)$ equals $\left(D^{1}, S^{0}\right)$ and $D^{1}$ is contractible. As there is a homotopy equivalence $S^{0} \wedge S^{0} \simeq S^{0}$, each $\widehat{A}^{I}$ is homotopy equivalent to $S^{0}$. Therefore there are homotopy equivalences

$$
\widehat{\mathbb{R Z}}_{K_{I}}:=\left({\widehat{D^{1}}, \underline{S^{0}}}^{K_{I}} \stackrel{\simeq}{\longrightarrow} \Sigma\left|K_{I}\right| \wedge \widehat{A}^{I} \simeq \Sigma\left|K_{I}\right| \wedge S^{0} \simeq \Sigma\left|K_{I}\right|\right.
$$

Thus the map $H$ becomes a homotopy equivalence

$$
H: \Sigma \mathbb{R} \mathcal{Z}_{K} \longrightarrow \bigvee_{I \subset[m]} \Sigma \mathbb{R} \mathcal{Z}_{K_{I}} \longrightarrow \bigvee_{I \subset[m]} \Sigma \widehat{\mathbb{R Z}}_{K_{I}} \stackrel{\simeq}{\longrightarrow} \bigvee_{I \subset[m]} \Sigma^{2}\left|K_{I}\right|
$$

It is in this form that we will use the Bahri, Bendersky, Cohen and Gitler decomposition because, as we will see shortly, it corresponds to a module decomposition of a differential graded algebra $R_{K}$ whose cohomology equals $H^{*}\left(\mathbb{R} \mathcal{Z}_{K}\right)$.

\section{Proof of the Main theorem}

Consider the following diagram

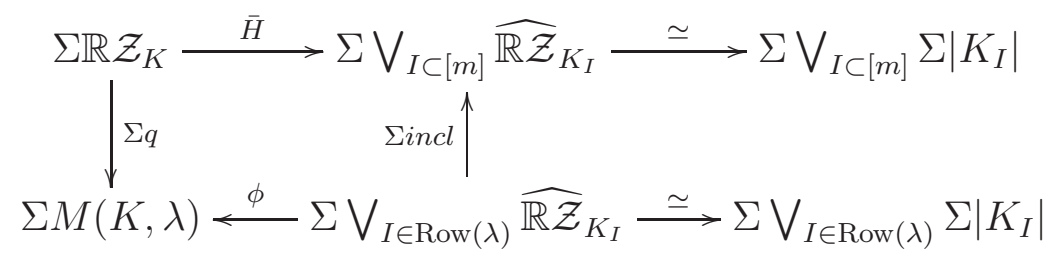

where, by definition, $\phi=\Sigma q \circ \bar{H}^{-1} \circ$ Eincl.

To prove Theorem 1.2 we will show that $\phi^{*}$ induces an isomorphism on cohomology. From now on, assume that coefficients in cohomology are $\mathbb{Q}$ or $\mathbb{Z}_{(p)}$, where $p$ is an odd prime.

First, by [5, Theorem 5.1] the cohomology ring of $\mathbb{R} \mathcal{Z}_{K}$ is given as follows. Let $\mathbb{Z}_{(p)}\left\langle u_{1}, \ldots, u_{m}, t_{1}, \ldots, t_{m}\right\rangle$ be the free associative algebra over the indeterminants of $\operatorname{deg} u_{i}=1, \operatorname{deg} t_{i}=0(i=1, \ldots, m)$. Define a differential graded algebra $R_{K}$ as the quotient

$$
R_{K}=\frac{\mathbb{Z}_{(p)}\left\langle u_{1}, \ldots, u_{m}, t_{1}, \ldots, t_{m}\right\rangle}{\left(u_{i}^{2}, u_{i} u_{j}+u_{j} u_{i}, u_{i} t_{i}-u_{i}, t_{i} u_{i}, t_{i} u_{j}-u_{j} t_{i}, t_{i}^{2}-t_{i}, t_{i} t_{j}-t_{j} t_{i}, u_{\sigma}(\sigma \notin K)\right)}
$$

where $i \neq j$ and $d\left(t_{i}\right)=u_{i}$ for each $i=1, \ldots, m$. Then $H^{*}\left(\mathbb{R} \mathcal{Z}_{K}\right) \simeq H^{*}\left(R_{K}\right)$. We shall use the notation $u_{\sigma}$ (respectively, $t_{\sigma}$ ) for the monomial $u_{i_{1}} \cdots u_{i_{k}}$ (respectively, $t_{i_{1}} \cdots t_{i_{k}}$ ) where 
$\sigma=\left\{i_{1}, \ldots, i_{k}\right\}, i_{1}<\cdots<i_{k}$, is a subset of $[m]$. For $I \subset[m]$, denote by $R_{K_{I}}$ the differential graded sub-module of $R_{K}$ spanned by the monomials $\left\{u_{\sigma} t_{I \backslash \sigma} \mid \sigma \in K_{I}\right\}$. Observe from the definitions of $R_{K}$ and $R_{K_{I}}$ that there is an additive isomorphism $R_{K}=\bigoplus_{I \subset[m]} R_{K_{I}}$.

Lemma 3.1. There is an additive isomorphism

$$
H^{*}\left(R_{K_{I}}\right) \simeq \tilde{H}^{*}\left(\widehat{\mathbb{R Z}}_{K_{I}}\right)
$$

and the projection $p_{I}: \mathbb{R Z}_{K} \rightarrow \widehat{\mathbb{R Z}}_{K_{I}}$ induces the inclusion $p_{I}^{*}: H^{*}\left(R_{K_{I}}\right) \hookrightarrow H^{*}\left(R_{K}\right)$.

Proof. The first assertion follows from $\widehat{\mathbb{R Z}}_{K_{I}} \simeq \Sigma\left|K_{I}\right|$ (see (3) ) and the isomorphism $H^{*}\left(R_{K_{I}}\right) \simeq$ $\tilde{H}^{*-1}\left(\left|K_{I}\right|\right)$ given by

$$
\begin{aligned}
R_{K_{I}} & \rightarrow C^{*}\left(K_{I}\right) \\
u_{\sigma} t_{I \backslash \sigma} & \mapsto \sigma^{*},
\end{aligned}
$$

where $C^{*}\left(K_{I}\right)$ is the simplicial cochain complex of $K_{I}$ ([5], Proposition 3.3]).

To show the second assertion, we look more closely at the isomorphism $H^{*}\left(R_{K}\right) \simeq H^{*}\left(\mathbb{R} \mathcal{Z}_{K}\right)$. From [5, §3.2], the monomials $u_{\sigma} t_{I \backslash \sigma}$ are mapped into the image of $p_{I}^{*}: C_{e}^{*}\left(\widehat{\mathbb{R Z}}_{K_{I}}\right) \rightarrow C_{e}^{*}\left(\mathbb{R} \mathcal{Z}_{K}\right)$, where $C_{e}^{*}$ denotes the cellular cochain complex. By combining this with the first assertion, we deduce the second assertion.

Now we investigate the maps appearing in (4). Since $\mid$ ker $\lambda \mid$ is a unit in the coefficient ring $\mathbb{Z}_{(p)}$, the map $q^{*}$ is injective with image $H^{*}\left(\mathbb{R}_{\mathcal{Z}_{K}}\right)^{\text {ker } \lambda}$. Notice that in cohomology incl induces the projection incl* $: \bigoplus_{I \subset[m]} H^{*}\left(R_{K_{I}}\right) \rightarrow \bigoplus_{I \in \operatorname{Row}(\lambda)} H^{*}\left(R_{K_{I}}\right)$. Recall that $\bar{H}=\Sigma \bigvee_{I \subset[m]} p_{I} \circ$ comul and $\phi=\Sigma q \circ \bar{H}^{-1} \circ \Sigma i n c l$. So $\phi^{*}$ is the composite

$\phi^{*}: H^{*}(\Sigma M(K, \lambda)) \simeq H^{*}\left(\Sigma \mathbb{R} \mathcal{Z}_{K}\right)^{\mathrm{ker} \lambda} \hookrightarrow H^{*}\left(\Sigma \mathbb{R} \mathcal{Z}_{K}\right) \stackrel{\simeq}{\rightarrow} \bigoplus_{I \subset[m]} H^{*}\left(\Sigma R_{K_{I}}\right) \rightarrow \underset{I \in \operatorname{Row}(\lambda)}{\bigoplus} H^{*}\left(\Sigma R_{K_{I}}\right)$,

where $\Sigma$ for graded modules means the degree shift in the positive degree parts.

We aim to show that $\phi^{*}$ is an isomorphism. To see this, we need two lemmas.

Lemma 3.2 ([9, Section 4]). The Reynolds operator

$$
N(x):=\frac{1}{|\operatorname{ker} \lambda|} \sum_{g \in \operatorname{ker} \lambda} g x
$$

induces an additive isomorphism $\bigoplus_{I \in \operatorname{Row}(\lambda)} R_{K_{I}} \stackrel{\simeq}{\rightarrow} R_{K}^{\mathrm{ker} \lambda}$, where $R_{K}^{\mathrm{ker} \lambda}$ is the ker $\lambda$-invariant ring of $R_{K}$. Furthermore, for a monomial $x=u_{\sigma} t_{I \backslash \sigma}, N(x)$ has the unique maximal term $x$, where the order is given by the containment of the index set.

Lemma 3.3. The composite

$$
\Phi: R_{K}^{\mathrm{ker} \lambda} \hookrightarrow R_{K} \simeq \bigoplus_{I \subset[m]} R_{K_{I}} \stackrel{\pi}{\rightarrow} \bigoplus_{I \in \operatorname{Row}(\lambda)} R_{K_{I}}
$$

is an isomorphism of rings, where $\pi$ is the projection. Note that we consider the ring structure of $\bigoplus_{I \in \operatorname{Row}(\lambda)} R_{K_{I}}$ induced by that of $R_{K}$.

Proof. We first show this is surjective. Take an element $x \in \bigoplus_{I \in \operatorname{Row}(\lambda)} R_{K_{I}}$. We induct on the size of the index set of $x$. By Lemma 3.2, the terms in $\pi(N(x)-x) \in \bigoplus_{I \in \operatorname{Row}(\lambda)} R_{K_{I}}$ has an index set strictly smaller than that for $x$. By induction hypothesis, there is an element $y \in R_{K}^{\mathrm{ker} \lambda}$ such that $\Phi(y)=\pi(N(x)-x)$. Put $z=N(x)-y \in R_{K}^{\mathrm{ker} \lambda}$ and we have $\Phi(z)=$ $\pi(N(x))-\Phi(y)=\pi(x)=x$.

On the other hand, suppose $\Phi(y)=0$ for some $y \in R_{K}^{\mathrm{ker} \lambda}$. By Lemma 3.2, there is $x \in$ $\bigoplus_{I \in \operatorname{Row}(\lambda)} R_{K_{I}}$ such that $y=N(x)$ and $y$ must contain the maximal terms in $x$. Thus, $\Phi(y)=0$ implies $x=0$ and $y=N(x)=0$. 
Proof of Theorem 1.2. Since $H^{*}\left(\mathbb{R} \mathcal{Z}_{K}\right)^{\operatorname{ker} \lambda} \simeq H^{*}\left(R_{K}^{\mathrm{ker} \lambda}\right)$, the definitions of $\phi$ and $\Phi$ imply that $\Sigma \Phi^{*}=\phi^{*}$. Therefore, by Lemma 3.3, $\phi^{*}$ is an isomorphism.

Corollary 3.4. We have the ring isomorphism

$$
H^{*}(M(K, \lambda)) \simeq H^{*}\left(\bigoplus_{I \in \operatorname{Row}(\lambda)} R_{K_{I}}\right)
$$

where the ring structure of the right hand side is induced by that of $R_{K}$.

\section{Stable Rigidity of ReAl toric Spaces}

In this section, we give an application of Theorem 1.2 to a stable rigidity property of real toric spaces.

Corollary 4.1. Let $M(K, \lambda)$ be a real toric space over $K$. When $\left|K_{I}\right|$ for any $I \in \operatorname{Row}(\lambda)$ suspends to a wedge of spheres after localization at an odd prime $p, \Sigma M(K, \lambda)$ is homotopy equivalent to a wedge of spheres after localization at $p$. Let $N\left(K^{\prime}, \mu\right)$ be another real toric spaces over $K^{\prime}$, where $\left|K_{I}^{\prime}\right|$ for any $I \in \operatorname{Row}(\mu)$ suspends to a wedge of spheres after localization at $p$. Then, if $H^{*}\left(M(K, \lambda) ; \mathbb{F}_{p}\right) \simeq H^{*}\left(N\left(K^{\prime}, \mu\right) ; \mathbb{F}_{p}\right)$ as modules, we have $\Sigma M(K, \lambda) \simeq_{p} \Sigma N\left(K^{\prime}, \mu\right)$.

Real toric spaces associated to graphs. Given a connected simple graph $G$ with $n+1$ nodes $[n+1]$, the graph associahedron $P_{G}([\underline{6}])$ of dimension $n$ is a convex polytope whose facets correspond to the connected subgraphs of $G$. Let $K$ be the boundary complex of $P_{G}$. We can describe $K$ directly from $G$ : the vertex set of $K$ consists proper subsets $T \subsetneq[n+1]$ such that $\left.G\right|_{T}$ are connected and the simplices are the tubings of $G$. We define a mod 2 characteristic map $\lambda_{G}$ on $K$ as follows:

$$
\lambda_{G}(T)= \begin{cases}\sum_{t \in T} \mathbf{e}_{t}, & \text { if } n+1 \notin T \\ \sum_{t \notin T} \mathbf{e}_{t}, & \text { if } n+1 \in T\end{cases}
$$

where $\mathbf{e}_{t}$ is the $t$-th coordinate vector of $\mathbb{F}_{2}^{n}$. Then we have a real toric manifold $M(G):=$ $M\left(K, \lambda_{G}\right)$ associated to $G$.

The signed a-number $s a(G)$ of $G$ is defined recursively by

$s a(G)= \begin{cases}1, & \text { if } G=\varnothing ; \\ 0, & \text { if } G \text { has a connected component with odd number of nodes } \\ -\sum_{T \subsetneq[n+1]} s a\left(\left.G\right|_{T}\right), & \text { otherwise, }\end{cases}$

and the a-number $a(G)$ of $G$ is the absolute value of $s a(G)$. As shown in [8], there is a bijection $\varphi$ from $\operatorname{Row}\left(\lambda_{G}\right)$ to the set of subgraphs of $G$ having an even number nodes and $\left|K_{I}\right|$ for $I \in \operatorname{Row}\left(\lambda_{G}\right)$ is homotopy equivalent to $\bigvee^{a(\varphi(I))} S^{|\varphi(I)| / 2-1}$ where $|\varphi(I)|$ is the number of nodes of $\varphi(I)$. By Theorem 1.2 we obtain the following.

Corollary 4.2. We have a homotopy equivalence

$$
\Sigma M(G) \simeq_{p} \bigvee_{I \in \operatorname{Row}\left(\lambda_{G}\right)} \bigvee^{a(\varphi(I))} S^{|\varphi(I)| / 2+1} \quad \text { for any odd prime } p
$$

Now, we define the $a_{i}$-number $a_{i}(G)$ of $G$ by

$$
a_{i}(G)=\sum_{\substack{T \subseteq[n+1] \\|\bar{T}|=2 i}} a\left(\left.G\right|_{T}\right) .
$$

Then, $a_{i}(G)$ coincides the $i$-th Betti number $\beta^{i}\left(M(G) ; \mathbb{F}_{p}\right)$ of $M(G)$. It should be noted that, by Corollary 4.2, if two graphs $G_{1}$ and $G_{2}$ have the same $a_{i}$-numbers for all $i$ 's, then $\Sigma M\left(G_{1}\right) \simeq_{p}$ $\Sigma M\left(G_{2}\right)$ for any odd prime $p$. 
Example 4.3. Let $P_{4}$ be a path graph of length 3 , and $K_{1,3}$ a tree with one internal node and 3 leaves (known as a claw). One can compute $a_{i}(G):=\sum_{\substack{T \subseteq[n+1] \\|T|=2 i}} a\left(\left.G\right|_{T}\right)$ as follows:

$$
\begin{aligned}
& a_{0}\left(P_{4}\right)=a_{0}\left(K_{1,3}\right)=1 \\
& a_{1}\left(P_{4}\right)=a_{1}\left(K_{1,3}\right)=3 \\
& a_{2}\left(P_{4}\right)=a_{2}\left(K_{1,3}\right)=2 \\
& a_{i}\left(P_{4}\right)=a_{i}\left(K_{1,3}\right)=0 \quad \text { for } i>2 .
\end{aligned}
$$

Hence, by Corollary $4.2, \Sigma M\left(P_{4}\right) \simeq_{p} \Sigma M\left(K_{1,3}\right)$ for any odd prime $p$ although $\Sigma M\left(P_{4}\right)$ and $\Sigma M\left(K_{1,3}\right)$ are not homotopy equivalent since they have different mod-2 cohomology.

Real toric spaces over fillable complexes. There is a wide class of simplicial complexes on which every real toric space satisfies the assumption in Corollary 4.1.

Definition 4.4 ([13, Definition 7.6]). Let $K$ be a simplicial complex. Let $K_{1}, \ldots, K_{s}$ be the connected components of $K$, and let $\hat{K}_{i}$ be a simplicial complex obtained from $K_{i}$ by adding all of its minimal non-faces. Then $K$ is said to be $\mathbb{F}_{p}$-homology fillable if (1) for each $i$ there are minimal non-faces $M_{1}^{i}, \ldots, M_{r}^{i}$ of $K$ such that $K_{i} \cup M_{1}^{i} \cup \cdots \cup M_{r}^{i}$ is acyclic over $\mathbb{F}_{p}$, and (2) $\hat{K}_{i}$ is simply connected for each $i$.

Moreover, we say that $K$ is totally $\mathbb{F}_{p}$-homology fillable when $K_{I}$ is $\mathbb{F}_{p}$-homology fillable for any $\emptyset \neq I \subset[m]$.

Proposition 4.5 ([13, Proposition 7.11]). If $K$ is $\mathbb{F}_{p}$-homology fillable, then $(\Sigma|K|)_{(p)}$ is a wedge of $p$-local spheres.

There is a large class of simplicial complexes which are totally homology fillable.

Proposition 4.6 ([13, Theorem 8.21]). If the Alexander dual of $K$ is sequentially CohenMacaulay over $\mathbb{F}_{p}([4])$, then $K$ is totally $\mathbb{F}_{p}$-homology fillable. $\mathbb{F}_{p}$.

Note that shifted and shellable simplicial complexes are sequentially Cohen-Macaulay over

\section{REFERENCES}

[1] A. Bahri, M. Bendersky, F. R. Cohen and S. Gitler, The polyhedral product functor: a method of decomposition for moment-angle complexes, arrangements and related spaces, Adv. Math. 225 (2010), no. 3, 1634-1668.

[2] G. Bredon, Introduction to Compact Transformation Groups, Pure and Applied Mathematics, vol. 46, Academic Press, New York-London, 1972.

[3] V. M. Buchstaber and T. E. Panov, Torus actions and their applications in topology and combinatorics, University Lecture Series, vol. 24, American Mathematical Society, Providence, RI, 2002.

[4] A. Björner, M. Wachs, and V. Welker, On sequentially Cohen-Macaulay complexes and posets, Israel J. Math. 169 (2009), 295-316.

[5] L. Cai, On products in a real moment-angle manifold, to appear in Journal of the Mathematical Society of Japan, arXiv:1301.1518.

[6] M. Carr and S. Devadoss, Coxeter complexes and graph-associahedra, Topology Appl. 153 (2006) 2155-2168.

[7] S. Choi, S. Kaji, and S. Theriault, Homotopy decomposition of a suspended real toric space, to appear in the special volume of the Boletin de la Sociedad Matemática Mexicana dedicated to Sam Gitler.

[8] S. Choi and H. Park, A new graph invariant arises in toric topology, J. Math. Soc. Japan, 67 (2015), no. 2, 699-720.

[9] S. Choi and H. Park, On the cohomology and their torsion of real toric objects, arXiv:1311.7056

[10] M. W. Davis and T. Januszkiewicz, Convex polytopes, Coxeter orbifolds and torus actions, Duke Math. J. 62 (1991), no. 2, 417-451.

[11] G. Denham and A. Suciu, Moment-angle complexes, monomial ideals and Massey products, Pure Appl. Math. Q. 3 (2007), 25-60.

[12] H. Ishida, Y. Fukukawa and M. Masuda, Topological toric manifolds, Moscow Math. J. 13 (2013), no. 1, 57-98. 
[13] K. Iriye and D. Kishimoto, The fat wedge filtration and a homotopy decomposition of a polyhedral product, arXiv:1412.4866v3

[14] L. Yu, On a class of quotient spaces of moment-angle complexes, arXiv:1406.7392,

Department of Mathematics, Ajou University, San 5, Woncheondong, Yeongtonggu, Suwon 443-749, KOREA

E-mail address: schoi@ajou.ac.kr

Department of Mathematical Sciences, Faculty of Science, Yamaguchi University, 1677-1, Yoshida, YAMAGUCHI 753-8512, JAPAN

E-mail address: skaji@yamaguchi-u.ac.jp

School of Mathematics, University of Southampton, Southampton SO17 1BJ, United KingDOM

E-mail address: S.D.Theriault@soton.ac.uk 